\title{
TAGUNG
}

\section{Innere und äußere Sicherheit als gemeinsame Aufgabe}

\author{
Frédéric Krumbein ${ }^{*}$
}

Die Europäische Kommission und das Europäische Parlament haben mit dem Vertrag von Lissabon neue Kompetenzen in den EU-Außenbeziehungen erhalten. Auf EU-Ebene zeichnet sich ein verändertes Verständnis von vernetzter „europäischer Außen- und Sicherheitspolitik" ab, das weit über die geläufige Auffassung einer Verschränkung ziviler und militärischer Kooperation hinausgeht. Verstärkt geht es in der europäischen Politik um eine institutionelle Verknüpfung von Innenund Justizpolitik mit dem auswärtigen Handeln der Europäischen Union.

Probleme wie internationaler Terrorismus, $\mathrm{Cy}$ berkriminalität, Migration oder der Zerfall staatlicher Strukturen sind grenzüberschreitende Probleme und bedürfen daher auch grenzüberschreitender Lösungsansätze. Bereits heute sind Aspekte der Innen- und Justizpolitik ein wesentlicher Bestandteil von Aktionsplänen in der Europäischen Nachbarschaftspolitik (ENP). Ihre Bedeutung für die Kooperation mit weiteren Drittstaaten nimmt zu. Die Innen- und Justizpolitik ist faktisch zu einem zentralen Bestandteil europäischer Außen- und Sicherheitspolitik geworden.

Im Mittelpunkt der Konferenz stand die Beantwortung von vier Fragen: Wie vollzieht sich der Prozess der Internationalisierung europäischer Innen- und Justizpolitik? Welche Rückwirkungen haben diese Prozesse wiederum auf die innen- und justizpolitischen Grundlagen europäischer Außen- und Sicherheitspolitik? Wie lösen die Europäische Union und ihre Mitgliedstaaten den Widerspruch auf zwischen der Einhaltung internationaler Rechtsstandards (Aufgabe der Innen- und Justizpoli-

\section{Die Innen- und Justizpolitik in der europäischen Außen- und Sicherheitspolitik}

Gemeinsame Tagung der Stiftung Wissenschaft und Politik und des Arbeitskreises Europäische Integration e.V.

Tagungsleitung: Dr. Ronja KEMPIN und Dr. Annegret BENDIEK

Mit finanzieller Unterstützung der Europäischen Kommission

Berlin, 9./10. Mai 2012

Begrüßung und kurze Einführung

Dr. Barbara LIPPERT, Stiftung Wissenschaft und Politik, Berlin

Die innere und äußere Sicherheit in der EU: Strukturen, Akteure und Inhalte

Reinhard PRIEBE, Direktor, Direktion Innere Sicherheit, Generaldirektion Inneres, Europäische Kommission, Brüssel

Discussant

Dr. Andreas MAURER, Stiftung Wissenschaft und Politik, Brüssel

Outside-In

Migration

Dr. Roderick PARKES, Stiftung Wissenschaft und Politik, Berlin

Cybercrime

Bundesministerium des Innern, Berlin

Nexus Failed States, OK und Terrorismus

Prof. Dr. Joachim KRAUSE, Universität Kiel

* Frédéric Krumbein, Geschäftsführer des Arbeitskreises Europäische Integration e.V., Berlin. 
tik) und der gleichzeitigen Unterminierung dieser Standards (durch die Außen- und Sicherheitspolitik)? Welche Rückschlüsse lassen sich für die Außen- und Sicherheitspolitik der Europäischen Union und ihrer Mitgliedstaaten ableiten? Ausgangspunkt der Konferenz waren nachfolgende Überlegungen über die Internationalisierung europäischer Innen- und Justizpolitik. (a) Grenzüberschreitende Probleme, wie Cyberkriminalität, Migration und Terrorismus setzen die Europäische Union zunehmend unter Handlungsdruck (Outside-In). (b) Diesen Herausforderungen versucht die Europäische Union eine Politik an den Außengrenzen oder in Drittstaaten entgegenzustellen, mit der sie den ,acquis communautaire' auf die Kooperationspartner zu übertragen hofft (Inside-Out). (c) Mit ausgewählten Kooperationspartnern, wie den USA und Russland, bemüht sich die Europäische Union um die Lösung von internationalen Problemen auf gleicher Augenhöhe (kooperative Regelsetzung).

\section{Europäische Schwerpunkte der inneren und äußeren Sicherheit}

Reinhard Priebe nannte gleich zu Beginn seines Vortrags die Schaffung des Raumes der Freiheit, der Sicherheit und des Rechts eine große Errungenschaft der europäischen Integration. Im ersten Teil seines Vortrags konzentrierte sich Priebe auf die Darstellung der Schwerpunkte der Politik der Europäischen Union im Bereich der inneren Sicherheit. Im zweiten Teil erläuterte er das Verhältnis der inneren und äußeren Dimension von Sicherheit.

Erster Schwerpunkt der europäischen Politik im Rahmen des Raumes der Freiheit, der Sicherheit und des Rechts stelle die Schwächung krimineller Netzwerke, wie im Bereich des Menschenhandels, dar. Das zweite Ziel bildeten Maßnahmen gegen den Terrorismus, zum Beispiel wie Geldflüsse von Terroristen effektiv überwacht werden könnten. Ein dritter Schwerpunkt sei der Bereich der Cybersicherheit und des Cyberkriminalität. In diesem neuen Feld bestehe eine große Heterogenität unter

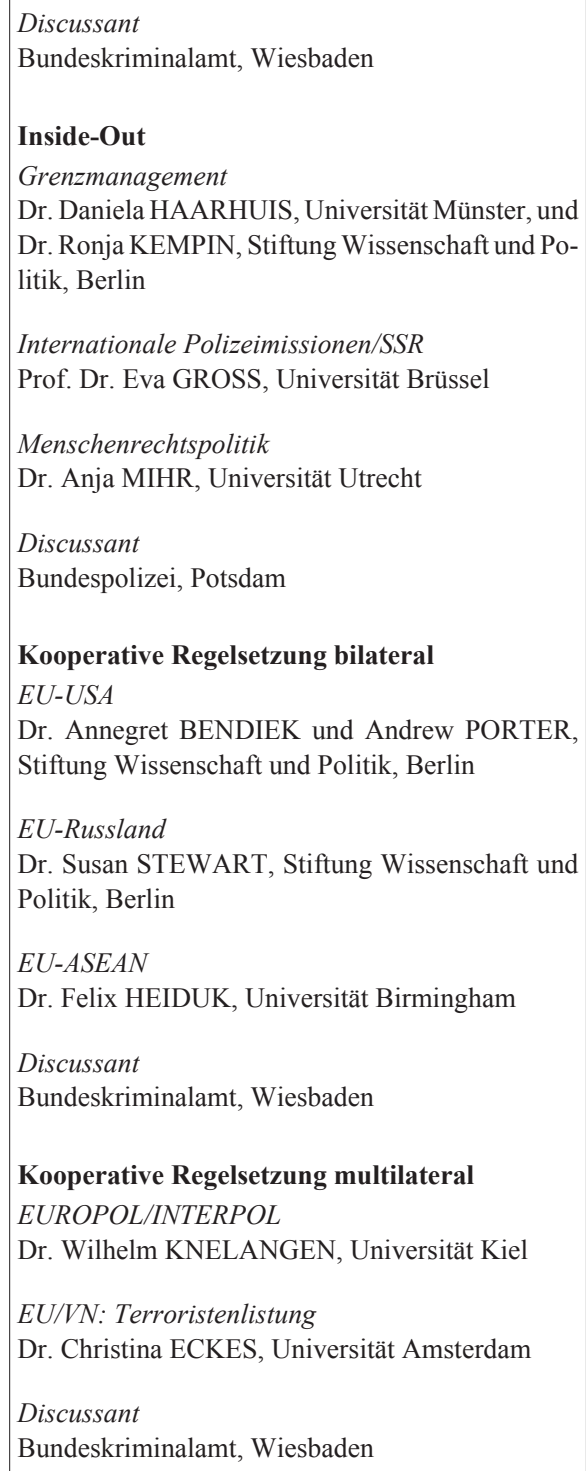

Dr. Daniela HAARHUIS, Universität Münster, und Dr. Ronja KEMPIN, Stiftung Wissenschaft und Politik, Berlin

Internationale Polizeimissionen/SSR

Prof. Dr. Eva GROSS, Universität Brüssel

Menschenrechtspolitik

Dr. Anja MIHR, Universität Utrecht

Discussant

Bundespolizei, Potsdam

\section{Kooperative Regelsetzung bilateral}

EU-USA

Dr. Annegret BENDIEK und Andrew PORTER, Stiftung Wissenschaft und Politik, Berlin

EU-Russland

Dr. Susan STEWART, Stiftung Wissenschaft und Politik, Berlin

EU-ASEAN

Dr. Felix HEIDUK, Universität Birmingham

Discussant

Bundeskriminalamt, Wiesbaden

Kooperative Regelsetzung multilateral

EUROPOL/INTERPOL

Dr. Wilhelm KNELANGEN, Universität Kiel

EU/VN: Terroristenlistung

Dr. Christina ECKES, Universität Amsterdam

Discussant

Bundeskriminalamt, Wiesbaden

den Staaten in den bereits getroffenen Maßnahmen.

Der vierte Schwerpunkt stelle die Verbesserung des Schutzes der Außengrenzen dar. Ein fünfter Schwerpunkt ziele auf die Herstellung der Handlungsfähigkeit der Europäischen Union nach Krisen, wie nach einer Naturkatastrophe oder einem Terroranschlag. 
Alle diese Schwerpunkte verfügten sowohl über eine Innen- als auch eine Außendimension. Die Europäische Union lege viel Wert darauf, die inneren und äußeren Dimensionen von Sicherheit gemeinsam zu betonen. Im Bereich der Cybersicherheit mache eine Unterscheidung zwischen innerer und äußerer Sicherheit am wenigsten Sinn.

Die Hauptsorge von Priebe galt der Tatsache, dass die Europäische Union oft zu schwerfällig agiere, wie bei der Verhandlung von Abkommen mit den USA im Antiterrorkampf (beispielweise das Fluggastdaten- $^{1}$ und das SWIFT-Abkommen). ${ }^{2}$ Komplizierte Abstimmungsprozesse bei Verhandlungen oder der Wunsch nach Transparenz der Verhandlungsprozesse von Seiten einzelner Abgeordneter des Europäischen Parlaments erschwerten die Politikgestaltung im Bereich der inneren Sicherheit.

Andreas Maurer ergänzte die rechtlichen Grundlagen mit Blick auf EU-Abkommen in der inneren und äußeren Dimension von Sicherheit. Er sehe drei Schnittmengen zwischen auswärtigem Handeln und der Innen- und Justizpolitik in der Europäischen Union. Die erste seien Antiterrorabkommen mit anderen Staaten, die sich auf die Resolution 1373 des Sicherheitsrates der Vereinten Nationen beriefen und von denen es etwa 20 Abkommen gebe. Die zweite stellten Abkommen mit Menschenrechtsklauseln dar, von denen es zurzeit 38 gebe. Die Klauseln würden Suspendierungen der Abkommen bei Menschenrechtsverletzungen vorsehen. Die dritte Kategorie bildeten Abkommen nach Art. 37 des Vertrags über die Europäische Union, wie Auslieferungsabkommen mit den USA und Japan oder Abkommen über die Weitergabe vertraulicher Informationen.

\section{Externe nichtmilitärische Bedrohungen der Europäischen Union}

Nach dem Überblick über die Schwerpunkte der Politik der Europäischen Union im Bereich der inneren Sicherheit und ihrer Verbindungen zum auswärtigen Handeln wurden in diesem Panel mögliche externe nichtmilitärische Bedrohungen der europäischen Sicherheit diskutiert, wie Cyberkriminalität oder Terrorismus.

Roderick Parkes zeichnete in seinem Vortrag über die Migrationspolitik der Europäischen Union das Bild einer imperialen und zuvörderst auf Sicherheit bedachten europäischen Politik. Sein Fokus war auf die Migrationspartnerschaften gerichtet, welche die Europäische Union mit Nachbarstaaten abgeschlossen hat, um den Migrationsdruck von den Außengrenzen der Europäischen Union abzuwenden. Die Europäische Union reagiere mit innen- und justizpolitischen Instrumenten auf eine externe Herausforderung. Sie knüpfe klare Bedingungen an die Vergabe der Mobilitätspartnerschaften. Sie „regiere" somit in die Staaten der unmittelbaren Nachbarschaft hinein. Gleichwohl dürfe auch hier nicht der Blick darauf verstellt werden, dass Mobilität und Migration auch als Chance für die Europäische Union und die Partnerstaaten zu begreifen seien. Für die Nachbarstaaten gingen die Partnerschaften mit Visaerleichterungen einher. Die Europäische Union könne die Migration nach Europa so bedarfsgerechter steuern.

Ein Vertreter des Bundesinnenministeriums referierte über Cyberkriminalität. Betrug und normale Kriminalität, die sich der Informationstechnologie bedienten, seien Teil der Cyberkriminalität. Im Jahr 2008 habe es 38.000 Fälle von der Cyberkriminalität gegeben, im Jahr 200950.000 und im Jahr 201060.000. Hinzu komme eine hohe Dunkelziffer. Cyberterrorismus gebe es bislang noch nicht.

1 Agreement between the United States of America and the European Union on the use and transfer of Passenger Name Records to the United States Department of Homeland Security.

2 Abkommen zwischen der Europäischen Union und den Vereinigten Staaten von Amerika über die Verarbeitung von Zahlungsverkehrsdaten und deren Übermittlung für die Zwecke des Programms der USA zum Aufspüren der Finanzierung des Terrorismus (TFTP). 
Die Innenministerkonferenz in Deutschland habe im Jahr 2010 eine Strategie zur Bekämpfung der Cyberkriminalität verabschiedet. Ziele der Strategie stellten die Kooperation mit der Wirtschaft bei der Bekämpfung der Cyberkriminalität, die Entwicklung spezialisierter Staatsanwaltschaften und die Gründung des Nationalen Cyber-Abwehrzentrums dar. Das Cyber-Abwehrzentrum diene der besseren Vernetzung verschiedener staatlicher Akteure.

Auf der europäischen Ebene existiere ein Übereinkommen über Computerkriminalität des Europarates aus dem Jahre 2001, das sogenannte Budapestabkommen. Weiterhin gebe es eine Richtlinie der Europäischen Union zur Vorratsdatenspeicherung ${ }^{3}$ und eine Richtlinie über Angriffe auf Informationssysteme. ${ }^{4}$ Auf der internationalen Ebene bestehe im Rahmen der ,G8“ eine Arbeitsgruppe und eine Zusammenarbeit im Rahmen von Interpol zum Thema Cyberkriminalität. Der Vertreter des Bundesministeriums des Innern sprach sich für Mindestspeicherfristen bei Telekommunikationsdaten aus. Seiner Ansicht nach könnten nur durch die Speicherung von Telekommunikationsdaten die Hintermänner von Terroristen und Kriminellen aufgedeckt werden.

Joachim Krause sprach über Unterschiede zwischen ,failed states', organisierter Kriminalität und Terrorismus. Schätzungen gingen davon aus, dass die organisierte Kriminalität zwischen 2 und 3 Prozent des globalen Bruttosozialproduktes ausmache. Terrorismus sei ein Sammelbegriff für die Anwendung von Gewalt, um politische Ziele zu erreichen. Das aktuell größte Problem stelle der islamische Terrorismus, wie beispielsweise al-Qaida, dar. Man vermute weltweit circa 120.000 bis 150.000 Kämpfer im Bereich des islamischen Terrorismus. Andere Formen existierten nach wie vor, wie separatistischer Terrorismus. ,Failed states' seien Staaten, in denen grundlegende staatliche Funktionen fehlten.

Eine These besage, dass ,failed states ' Terrorismus verursachten. Eine weitere These sehe die Bildung einer Allianz von Terrorismus und organisierter Kriminalität. Beide Thesen würden jedoch nicht zutreffen. Schnittstellen zwischen ,failed states', Terrorismus und organisierter Kriminalität existierten, aber die Protagonisten der organisierten Kriminalität und des Terrorismus seien sehr verschieden und hätten unterschiedliche Ziele. Terroristen verfolgten meist nicht materielle, sondern idealistische Ziele. Die kurdische separatistische Terrororganisation PKK stelle ein Beispiel für eine enge Verknüpfung zwischen Terrorismus und organisierter Kriminalität dar. So pflanze die PKK Drogen an und exportiere diese. Kriminelle und Terroristen verfügten über unterschiedliche Ziele, aber ähnelten sich in den Strukturen und hätten teilweise die gleichen Gegner. Viele Autoren stimmten darin überein, dass die organisierte Kriminalität oftmals die Grundlage für die Finanzierung terroristischer Organisationen sei.

Seit 2001 sei eine Zunahme der Kooperation zwischen Terroristen und organisierter Kriminalität festzustellen. Die Finanzierungsquellen für Terroristen hätten durch den Antiterrorkampf der USA seit dem Jahr 2001 drastisch abgenommen. Es stelle sich die Frage, ob der islamische Terrorismus sich in Zukunft stärker der organisierten Kriminalität annähern werde oder nicht. Die organisierte Kriminalität brauche vor allem reiche Staaten, weil dort ihre ,Kunden' wohnten und die Profite nur dort dauerhaft gesichert werden könnten. ,Failed states' stellten eher Rückzugsorte dar, in denen Strafverfolgungsbehörden keinen Zugriff hätten.

3 Richtlinie 2006/24/EG des Europäischen Parlaments und des Rates vom 15. März 2006 über die Vorratsspeicherung von Daten, die bei der Bereitstellung öffentlich zugänglicher elektronischer Kommunikationsdienste oder öffentlicher Kommunikationsnetze erzeugt oder verarbeitet werden, und zur Änderung der Richtlinie 2002/58/ EG, in: Amtsblatt der EU, Nr. L 105 vom 13. April 2006, S. 54-63.

4 Europäische Kommission: Vorschlag für eine Verordnung des Europäischen Parlaments und des Rates über die elektronische Identifizierung und Vertrauensdienste für elektronische Transaktionen im Binnenmarkt, KOM (2012) 238 . 
Cyberkriminalität war das Thema des Vortrags eines Vertreters des Bundeskriminalamtes. Dieses Thema sei im Moment „en vogue“ und viele Staaten wünschten sich Unterstützung beim Aufbau von Zentren zur Bekämpfung von Cyberkriminalität.

Der Vertreter des Bundeskriminalamtes stimmte Krause zu, dass die vorher erwähnten Thesen falsch seien: ,Failed states' stellten nicht die Ursache von Terrorismus und organisierter Kriminalität dar und Terrorismus und organisierte Kriminalität verbündeten sich nicht flächendeckend. Das Zusammenspiel von terroristischen und kriminellen Organisationen könne mit der Zusammenarbeit zwischen verschiedenen Unternehmen verglichen werden. Zum Beispiel bauten einige terroristische Organisationen Drogen an und verwendeten Netzwerke organisierter Kriminalität für den Vertrieb.

\section{Die EU im Spannungsfeld von Grenzsicherung und Achtung der Menschenrechte}

Das Spannungsfeld zwischen dem Streben nach Sicherheit und der Förderung freiheitlicher Wertvorstellungen wurde im zweiten Panel thematisiert. Die Achtung der Menschenrechte als einer der Grundpfeiler der europäischen Werteordnung bildet den roten $\mathrm{Fa}$ den. Doch dieser sei nicht immer sichtbar und Widersprüche zwischen der Verletzung der Menschenrechte durch EU-Organe und EUMitgliedstaaten bei der Sicherung der gemeinsamen Außengrenzen bei gleichzeitiger Förderung der Menschenrechte in der EU-Außenpolitik bedürften daher der Diskussion.

Ronja Kempin führte aus, wie durch die schrittweise Abschaffung der Binnengrenzen mit dem Vertrag von Amsterdam der Schutz der EU-Außengrenzen eine größere Bedeutung erhalten hat und die Agentur Frontex geschaffen wurde. Über Frontex übertrage die Europäische Union durch die Ausbildung von Personal sowie durch ,working agreements" Teile des ,acquis communautaire' auf Drittstaaten. Drittstaaten müssten EU-Werte, -Standards und -Instrumente übernehmen, um mit ihr im Bereich des Grenzmanagements kooperieren zu können.

Daniela Haarhuis führte ergänzend den menschenrechtlichen Aspekt des Grenzmanagements aus. Die Europäische Union versuche, die Grenzen „vorzuverlegen“, um schon in Nordafrika viele Flüchtlinge an der Reise nach Europa zu hindern. Einige Abgeordnete des Europäischen Parlaments, wie die Vorsitzende des Menschenrechtsausschusses Barbara Lochbihler, betrachteten die Arbeit von Frontex als Verstoß gegen die Menschenrechte. In der Tat gebe es berechtigte Zweifel daran, ob die Europäische Union ihren eigenen Standards immer gerecht werde. So gab der Europäische Gerichtshof für Menschenrechte einer Klage gegen Italien statt, das Flüchtlinge nach Libyen ausgewiesen hatte, ohne die Menschenrechtssituation in Libyen und die Ansprüche der Flüchtlinge auf Asyl zu prüfen. Das Grenzmanagement der Europäischen Union werde somit in einigen Fällen den eigenen menschenrechtlichen Standards nicht gerecht. Diese Darstellung wurde von einem Vertreter der Bundespolizei mit dem Hinweis korrigiert, dass die Verantwortung für die Grenzsicherung bei den jeweiligen Staaten liege und Frontex nur assistiere. Die Agentur arbeite mit dem Hohen Flüchtlingskommissar der Vereinten Nationen (UNHCR) zusammen, um den Flüchtlingen optimal zu helfen. Frontex müsse auch die Staaten bei Ermittlungsverfahren unterstützen, um beispielsweise kriminelle Schleuserbanden langfristig auszuschalten. Die Aufgaben von Frontex bestünden vorrangig darin, die Mitgliedstaaten zu motivieren und zu unterstützen, ihre Grenzschutzaufgaben effektiv wahrzunehmen.

Anja Mihr referierte über die Menschenrechtspolitik der Europäischen Union. Menschenrechtspolitik stelle primär eine Aufgabe der europäischen Außenpolitik dar. Die Europäische Union bemühe sich zunehmend um eine stärkere Kohärenz und eine Verzahnung zwischen der inneren und der äußeren Dimension von Menschenrechtspolitik. Die Men- 
schenrechtspolitik, vor allem ihre Glaubwürdigkeit, hänge stark von der Umsetzung der Menschenrechte in den einzelnen Staaten der Europäischen Union ab.

Bislang gebe es keine Indikatoren und ,benchmarks' zur Bewertung des Erfolgs der europäischen Menschenrechtspolitik. Schwerpunkte seien unter anderem die Abschaffung der Todesstrafe, das Verbot von Folter und das Verbot der Diskriminierung von Frauen. Alle Schwerpunkte fänden sich in acht „human rights guidelines" der Europäischen Union. Bei der Todesstrafe lasse sich relativ leicht der Rückgang von Exekutionen als ein Indikator für Fortschritte der EU-Politik verwenden.

Das Europäische Instrument für Menschenrechte und Demokratie sei ein wichtiges Instrument der EU-Politik. Die Europäische Union könne künftig auch Vertragspartei von internationalen Menschenrechtsverträgen werden.

Die Fähigkeit der Europäischen Union, eigene Wertvorstellungen an Drittstaaten zu vermitteln, wurde von Eva Gross infrage gestellt. Ihr Beitrag richtete den Blick auf die Bemühungen der Union, den Sicherheitssektor in schwachen oder gescheiterten Staaten zu reformieren. Obgleich die Mitgliedstaaten die Reform des Sicherheitssektors als einen zentralen Pfeiler der Gemeinsamen Sicherheits- und Verteidigungspolitik (GSVP) identifiziert hätten, fehle es den entsprechenden Missionen bislang sowohl an geschultem Personal als auch an finanziellen Mitteln und einer mittel- bis langfristigen Strategie. Eine detaillierte Analyse bisheriger EU-Missionen, wie etwa Eupol Afghanistan zum Aufbau der Afghanischen Nationalen Polizei, zeige die Schwächen der Union überdeutlich. Der Mitteleinsatz der Europäischen Union reiche bei Weitem nicht an den der USA heran. Noch immer sei die 400 Experten umfassende Mission personell nicht in vollem Umfang handlungsfähig. Darüber hi- naus verfolgten die Mitgliedstaaten nationale Polizeiausbildungsprogramme oder stellten ihre Experten der NATO-Mission, ${ }^{5}$ nicht aber der Europäischen Union zur Verfügung. Im Ergebnis bestimmten die USA und nicht länger die Europäische Union Dauer und Inhalte der Ausbildungsmodule. Zudem vernachlässige die Europäische Union den Auf- und Ausbau eines funktionierenden Justizwesens - Grundlage sinnvoller Polizeiarbeit - vollständig. Eine Verzahnung von Innen- und Justiz- sowie Außen- und Sicherheitspolitik finde in den zivilen Missionen der GSVP bislang kaum statt. Daher sei es der Europäischen Union auch nicht gelungen, ihre Normen, Werte und Standards eines funktionierenden Sicherheitsapparats erfolgreich auf Drittstaaten zu übertragen.

\section{Kooperation der EU mit den USA, Südostasien und Russland}

Das dritte Panel widmete sich der Kooperation der Europäischen Union im Bereich der nichtmilitärischen Sicherheit mit anderen Staaten und Staatengruppen. Hier wurde aufgezeigt, dass auch in diesem Bereich eine enge Kooperation mit dem traditionellen Verbündeten USA besteht. Die Kooperation mit der ASE$\mathrm{AN}^{6}$ und Russland beschränkt sich hingegen auf politische Dialoge sowie Visa- und Handelsfragen.

Annegret Bendiek und Andrew Porter referierten über die Kooperation zwischen der Europäischen Union und den USA im Bereich der Cybersicherheit. Cyberangriffe auf kritische Infrastrukturen und auf Regierungsinstitutionen seien zentrale Herausforderungen, die in der Zusammenarbeit thematisiert und für die Abwehrmaßnahmen entwickelt würden. Prioritäten der Europäischen Union seien, Robustheit und Reaktionsfähigkeit von Cybersicherheit sowie Internetsicherheit zu stärken, private Akteure einzubeziehen und globale Kooperationen im Bereich der Cybersicherheit einzugehen. Die Strategie der Cybersicherheit der

5 North Atlantic Treaty Organization.

6 Association of Southeast Asian Nations. 
USA basiere auf mehreren Zielen, die denen der Europäischen Union ähnelten: Prävention, Schutz, ,resilience“ und internationale Kooperation. Im Bereich der US-EU-Kooperation gebe es seit 2010 eine Arbeitsgruppe zu Cybersicherheit. Im Rahmen der NATO existiere seit 2008 ein „NATO Cooperative Cyber Defence Centre of Excellence“. Das Budapestabkommen des Europarates von 2001 sei noch der zentrale Vertrag auf europäischer, transatlantischer und globaler Ebene zur Bekämpfung von Cyberkriminalität. Auf der internationalen Ebene stelle die „International Telecommunication Union" das zentrale multilaterale Forum im Bereich der Cybersicherheit dar. Die transatlantische Kooperation in der Cybersicherheit wird zusammenfassend als eng und vertrauensvoll evaluiert.

Eindrückliche Beispiele für die enge Kooperation seien die inzwischen regelmäßig zwischen EU-Staaten und den USA stattfindenden Übungen zur Abwehr von Cyberangriffen. Auch die Zusammenarbeit zwischen Deutschland und den USA im Bereich Cyberkriminalität verlaufe sehr gut. So gebe es vom USamerikanischen Federal Bureau of Investigation und vom Secret Service, den beiden Dienststellen, mit denen Deutschland in diesem Bereich kooperiere, Verbindungsbeamte im US-Konsulat in Frankfurt. Ein Problem bleibe, dass Rechtshilfeersuchen zwischen den USA und Deutschland etwa zwischen vier und acht Monaten dauerten. Dies sei deutlich zu lang vor dem Hintergrund der Schnelllebigkeit von Internetverbrechen.

Susan Stewart sprach über die Kooperation zwischen der Europäischen Union und Russland. Seit 1994 gebe es ein gemeinsames Abkommen. Dieses werde gerade neu verhandelt. Hinzu kämen sogenannte Gemeinsame Räume, wie im Bereich Wirtschaft, für die teilweise gemeinsame Strategien existierten und die unterschiedlich gut umgesetzt würden. Ein Gemeinsamer Raum bestehe ebenfalls im Bereich Freiheit, innere Sicherheit und Justiz. Dieser Raum sei thematisch sehr weit gefasst und beinhalte beispielsweise Grenzmanagement und
Migration. Wertefragen würden von Russland eher verdrängt. Damit werde eines der Ziele der Europäischen Union, die Förderung der eigenen Werte in Drittstaaten, konterkariert.

In der Praxis nehme das Ziel eines visafreien Raumes den größten Platz innerhalb des Gemeinsamen Raumes der Freiheit, der Sicherheit und des Rechts ein. Hier sei es auch schon $\mathrm{zu}$ Visaerleichterungen gekommen. In anderen Bereichen gebe es nur wenige Fortschritte. Überwiegend hätten Dialoge und Gespräche stattgefunden. Weiterhin gebe es Menschenrechtskonsultationen, die aber zu keinen Fortschritten führten. Die Europäische Union mache Vorschläge und liste Probleme auf, ohne dass sich in Russland etwas ändere. Russland hätte es geschafft, die eigenen Prioritäten in den Verhandlungen auf die Tagesordnung zu setzen, wie die Visafreiheit. Wirtschaftsfragen stünden im Vordergrund der Beziehungen. Die gewünschte Visafreiheit diene ebenfalls in erster Linie dem wirtschaftlichen Austausch.

Die Kooperation der Europäischen Union mit der ASEAN im Bereich des Antiterrorkampfes war das Thema des Vortrags von Felix Hei$d u k$. Außer Dialogforen gebe es kaum eine $\mathrm{Zu}$ sammenarbeit in diesem Bereich. Generell stehe der Handel zwischen der Europäischen Union und der ASEAN im Vordergrund der Beziehungen. Im politischen Bereich sei der Konflikt um den Umgang mit Myanmar ein Hemmschuh für den weiteren Ausbau der Beziehungen gewesen.

Seit dem terroristischen Anschlag auf Bali im Jahr 2002 sei der Antiterrorkampf stärker in den Fokus gerückt. Islamischer Terrorismus spiele in Südostasien eine Rolle. Seit dem Anschlag auf Bali habe es mehrere weitere terroristische Anschläge auf westliche Ziele in Südostasien gegeben. Das zentrale Ziel der Europäischen Union in der Region stelle der Schutz von EU-BürgerInnen und der Schutz der Seewege, wie der Straße von Malakka, dar. Mehrere Deklarationen, wie die ,Joint Declaration on Co-operation to Combat Terrorism“ "der Europäischen Union und der ASEAN von 2003, 
seien seit dem Anschlag auf Bali 2002 verabschiedet worden. Diese enthielten aber keine Aktionspläne oder konkreten Maßnahmen, sondern stellten in erster Linie Absichtserklärungen dar.

Im Jahr 2007 verabschiedeten die ASEAN und die Europäische Union die Nürnberger Erklärung, die fünf Kooperationsbereiche nenne, darunter eine Kooperation über Sicherheitsfragen. Hier habe es zum ersten Mal einen Aktionsplan gegeben. Trotzdem bliebe der Dialog die Hauptaktivität im Bereich der Bekämpfung des Terrorismus.

Drei Gründe stünden einer engeren Zusammenarbeit im Wege. Erstens, der „ASEAN way", spiele eine Rolle. Wahrung und Achtung der nationalen Souveränität sei ein zentraler Wert der südostasiatischen Staaten und erkläre sich durch die Erfahrungen mit dem Kolonialismus in der Region. Neutralität, friedliche Konfliktbeilegung und konsensuale Entscheidungsprozesse stellten weitere Werte dar, die einer engeren Kooperation im Wege stünden. Zweitens, der Umgang der ASEAN-Mitglieder mit dem Problem Terrorismus behindere eine engere Zusammenarbeit. Terrorismus werde von den ASEAN-Staaten nur als eines von vielen Sicherheitsproblemen wahrgenommen. Das Problem der zwischenstaatlichen Sicherheit bleibe für sie zentral. Hinzu komme, dass viele Staaten Terrorismus unter expliziter Nichtbeachtung der Menschenrechte bekämpften. Drittens, die marginale politische Rolle der Europäischen Union in Südostasien behindere eine stärkere Kooperation. Die USA blieben der zentrale sicherheitspolitische Akteur und der Aufstieg Chinas bestimme die Überlegungen und Politiken vieler ASEANStaaten.

Insgesamt bestehe die EU-ASEAN-Kooperation im Bereich Terrorismus vor allem in Deklarationen und Dialogen. In den gemeinsamen Erklärungen fänden sich sowohl die Werte und Normen der Europäischen Union als auch die der ASEAN, zwischen denen teilweise ein Spannungsverhältnis bestünde.
Ein Vertreter des Bundeskriminalamtes sprach über Cyberkriminalität. Für das Jahr 2010 habe man 250.000 Straftaten unter Verwendung des Internets registriert. Straftaten, die im engeren Sinne als Cyberkriminalität bezeichnet würden, habe es 60.000 gegeben. Der Trend gehe dahin, dass sich die kriminellen Strukturen zunehmend national und international vernetzten und nicht nur hochspezialisierte und technisch versierte kleine Gruppen in diesem Bereich agierten. Die Aufklärungsquote liege im Bereich der Cyberkriminalität bei nur 30 Prozent, während sie insgesamt etwa 50 Prozent betrage.

Cybersicherheit sei in erster Linie eine Frage der technischen Sicherheit und nicht der individuellen. Viele Verbrechen im Internet würden nicht als Verbrechen betrachtet oder ausschließlich als Bagatelldelikte eingestuft. Es fehle das öffentliche Bewusstsein für diese Form der Kriminalität und die Schwere der Straftaten.

Annegret Bendiek ergänzte in der Diskussion, dass es bislang kaum Regeln für das Internet gebe. Einzig die „Budapestkonvention“ existiere in diesem Bereich. Die Konvention sei aber nicht umfassend ratifiziert und man befinde sich noch ganz am Anfang eines Regelsetzungsprozesses.

\section{Kooperation der EU mit internationalen Organisationen}

Im vierten Panel berichteten die Referenten über die Zusammenarbeit von EU-Organen mit den internationalen Organisationen Interpol und den Vereinten Nationen.

Wilhelm Knelangen zufolge sei Interpol von PraktikerInnen gegründet worden und international nur schwach rechtlich verankert. Dies habe aber auch viele Vorteile bei der praktischen Kooperation und der Strafverfolgung. Interpol und Europol unterstützten die nationalen Polizeibehörden in ihrer Arbeit. Interpol sei ein Übermittlungsweg für den Informationsaustausch zwischen den Mitgliedstaaten. 
Inzwischen existiere eine internetbasierte Datenbank.

Bei Europol gebe es ein Informationssystem für Mitgliedstaaten und Europol erstelle gemeinsame Analysen, die der Verbrechensbekämpfung dienten. Europol sei eher ein kleiner Akteur im Vergleich mit den nationalen koordinierenden Strafverfolgungsbehörden.

Europol habe im Rahmen der EU-Erweiterung auch Kooperationsabkommen geschlossen, um den neuen Mitgliedstaaten bei der Übernahme des, acquis communautaire' im Bereich Europol zu helfen. Weitere Kooperationsabkommen bestünden mit Nachbarstaaten der Europäischen Union und anderen Drittstaaten, wie Australien oder Kolumbien, und mit Interpol. Eine wichtige Voraussetzung stelle ein bestimmtes Niveau des Datenschutzes in den Kooperationsstaaten dar.

Es gebe vier konkurrierende Modelle vom gegenwärtigen Europol und seiner Rolle bei der Normverbreitung. Das erste Modell sehe Europol als ein effizientes Organ der Verbrechensbekämpfung. Das zweite Modell sehe Europol als ein Organ der multilateralen Polizeikooperation. Eine möglichst umfassende Kooperation mit vielen Partnern stehe im Vordergrund. Im dritten Modell stehe Europol im Zentrum der Idee einer demokratischen und rechtsstaatlichen Polizeiarbeit. Das bedeute, dass die Partner ebenfalls demokratische und rechtsstaatliche Normen einhalten müssten. Das vierte Modell ordne Europol als eine Behörde ein, die sich auf ,,intelligence“, das heißt die Sammlung und Auswertung von Daten stütze. Die Polizeiarbeit werde damit mehr proaktiv und bleibe nicht nur reaktiv. Die vier Modelle würden sich nicht ausschließen, sondern beschrieben nur unterschiedliche Facetten der Arbeit von Europol und der Zusammenarbeit mit verschiedenen Partnern.

Das Interesse für eine Kooperation zwischen Interpol und Europol bestehe in Synergieeffekten. Europol gewinne zusätzliche Daten und könne Kontakte mit internationalen Part- nern knüpfen. Für den Bereich Menschenrechte gebe es eine Vereinbarung zwischen Europol und Interpol, die den Schutz der Daten von Europol sicherstelle. Europol sei aber insgesamt zurückhaltend, die Einhaltung von Menschenrechten in den Beziehungen zu Interpol $\mathrm{zu}$ thematisieren. Nicht wenige Polizeibehörden, die bei Interpol mitarbeiten, verletzten Menschenrechte.

Christina Eckes referierte über Sanktionen gegen Terrroristen und die rechtlichen Schwierigkeiten in diesem Bereich, beispielsweise bei der Zusammenarbeit zwischen den Vereinten Nationen, der Europäischen Union und den Mitgliedstaaten.

Seit 1999 gebe es Antiterrorismussanktionen gegen Personen, die auf den Resolutionen 1267 und 1373 des Sicherheitsrates der Vereinten Nationen basierten. Die Vereinten Nationen definierten teilweise wer Terrorist sei, teilweise tue die Europäische Union dies selbst. Das hänge von der Art und der Herkunft der Terroristen ab. Bei den Vereinten Nationen gebe es Listen von Terroristen. Inzwischen existiere eine Ombudsperson, die über Beschwerden von Personen entscheide, die sich auf der Liste befänden. Früher habe es keine Möglichkeit der Beschwerde gegen eine Listung gegeben.

Ein Problem der Liste von Terroristen bestehe in der Geheimhaltung. So wüssten oftmals nur die Staaten oder Organisationen, die eine Person auf eine Liste von Terroristen setzten, warum sie diese Entscheidung getroffen haben. Andere Staaten hätten vielfach keinen Einblick in die Gründe für die Entscheidungen und seien nicht in der Lage, diese nachzuvollziehen. Rechtsschutz für einzelne Personen auf Listen von Terroristen stellten vor allem Gerichte her. Allerdings sei der Rechtsschutz sehr heterogen ausgeprägt. Der Europäische Gerichtshof setze und garantiere einen höheren Rechtsschutz als viele der EU-Mitgliedstaaten.

Eine Vertreterin des Bundeskriminalamtes kommentierte in erster Linie den Vortrag von Knelangen. Interpol sei vor allem ein Informa- 
tionsforum und offiziell ein Verein nach französischem Vereinsrecht, in dem die nationalen Polizeibehörden Mitglied sind und nicht die Staaten. Interpol sei eine politisch neutrale Organisation. Deutschland liefere Daten nur eingeschränkt an Interpol, weil Deutschland sich davor fürchte, dass die Daten missbraucht werden könnten. Viele Staaten bei Interpol verfügten allerdings auch nur über eingeschränkten Zugang zu Daten.

Die Kapazitäten von Interpol seien begrenzt. So sei die Fingerabdruckdatenbank Deutschlands deutlich größer als die von Interpol. Interpol werde nur von wenigen Mitgliedstaaten finanziert. Die anderen profitierten überwiegend von Unterstützung durch Interpol, zum Beispiel durch Ausbildungsmaßnahmen für die eigenen Polizisten.

Insgesamt wandelten die EU-Staaten auf einem schmalen Grat zwischen der Vertretung der Einhaltung von Menschenrechtsnormen bei Interpol und der Effektivität der Arbeit der Institution. Die Zusammenarbeit von Interpol mit autoritären Regimen, wie im Fall Belarus, gehöre zu den strittigsten Themen innerhalb von Interpol.

\section{Der Schutz der Menschenrechte als Herausforderung von innerer und äußerer Sicherheitspolitik}

Die Tagung beleuchtete die Verbindung von innerer und äußerer Sicherheit der Europäischen Union aus unterschiedlichen Sichtweisen: von PraktikerInnen nationaler und europäischer Behörden und von WissenschaftlerInnen unterschiedlicher Disziplinen.

Diese fruchtbare Kombination zeigte im ersten Teil der Tagung gegenwärtige Bedrohungen der inneren Sicherheit auf. In der Bewertung der Bedrohungen herrschte weitgehende Einigkeit unter den ReferentInnen und TeilnehmerInnen.
In der Diskussion der äußeren Dimension von Sicherheit zeigten sich - wie oft bei Themen mit Bezug zur Europäischen Union - die teilweise beschränkten Handlungsmöglichkeiten europäischer Organe und die gelegentlich schwerfälligen Prozesse in der europäischen Politikformulierung und -implementierung.

Im dritten und vierten Teil wurde deutlich, dass sich die Europäische Union in der Kooperation mit externen Akteuren - mit Ausnahme der USA - oftmals schwer tut. Teilweise fehlen die institutionellen Kapazitäten und Kompetenzen, wie bei Europol, teilweise fehlt das Interesse und die Kohärenz auf Seiten der Europäischen Union und/oder ihrer Partner, wie bei der ASEAN und Russland.

Ein Defizit der Tagung zeigte sich in der eingeschränkten Diskussion der Einhaltung der Menschenrechte im Bereich der inneren und äußeren Sicherheit. Hier fehlten bei der Tagung die Akteure, die gewissermaßen als ,Hüter' dieser Normen auftreten: Menschenrechtsorganisationen und die Justiz. Der Bereich der Grenzsicherung, bei dem vielfältige Menschenrechtsverletzungen der Organe der Europäischen Union und ihrer Mitgliedstaaten erfolgen, wurde vor allem aus dem Blickwinkel der europäischen Akteure und nicht aus der Perspektive der Flüchtlinge betrachtet. Menschenrechtsnormen werden im Sicherheitsbereich zu wenig beachtet. Dies gilt vor allem für den Bereich der Grenzsicherung und der Kooperation der Europäischen Union und ihrer Mitgliedstaaten mit anderen Staaten, wie im Bereich des Antiterrorkampfes.

Insgesamt besteht in der Europäischen Union die Notwendigkeit, innere und äußere Sicherheit stärker zu verschränken. Dies betrifft sowohl die rechtlichen und institutionellen Grundlagen, wie dem Primärrecht, als auch die praktische Politik der Europäischen Union. 